\title{
ortwin Renn Akzeptanzforschung: Technik in der gesellschaftlichen Auseinandersetzung
}

Als die Meinungsforscher des Instituts für Demoskopie in Allensbach 1968 durch die Lande reisten, um weibliche Bundesbürger zu befragen, welchen Beruf wohl ihr Traummann haben solle, schnitt eine Berufsgruppe am besten ab: der Atomphysiker. Elf Jahre später stellten die Demoskopen dieselbe Frage: Welcher Beruf gefällt Ihnen besonders für einen Mann, welchen Beruf sollte Ihr Traummann haben? Bei dieser erneuten Befragung wurde der Atomphysiker weit abgeschlagen. An erster Stelle stand nun - der Förster, Inbegriff der grünen Idylle, zumindest vor dem erst dann einsetzenden Waldsterben.

So wenig man mit dieser - eher flapsigen Fragestellung - wissenschaftliche Analysen über die Stellung der Atomphysik in der Bundesrepublik Deutschland anstellen kann, so sehr illustriert dieser Wandel im Bild des idealen Ehepartners die Veränderung des Zeitgeistes und der gängigen Wertvorstellungen. Galt der Atom- und Kernphysiker noch vor 15 Jahren als Hort des glückverheißenden Fortschritts, als Übermittler der Faszination befreiender Technik und als Inbegriff der Emanzipation zur Wohlstandsgesellschaft, so verkörpert er 15 Jahre später den Advokaten einer verselbständigten Großtechnologie, den Anhänger einer ökologischen Ausbeutungsideologie und den Handlanger finsterer Kapitalverwertungsinteressen.

$\mathrm{Da}$ sich die Vorstellungen über Technik und ihre Folgen im Laufe der Zeit wandeln, ist offenkundig. Für die politische Entscheidungsfindung über den Einsatz von Technologien ist es von besonderem Interesse, wie die Bürger auf neue Technologien reagieren. Denn die Ausbreitung neuer Technologien ist weitgehend davon abhängig, daß in der Gesellschaft technische Neuerungen als Bereicherung des sozialen Lebens begriffen werden. Übersetzt in soziologische Terminologie heißt das: Die Veränderungen in Technik und Produktion müssen auf Akzeptanz stoßen.

Dabei bedeutet Akzeptanz mehr als passive Duldung der ,von oben " verordneten technischen Neuerungen. Vielmehr soll hier unter Akzeptanz die positive Aufnahme einer Veränderung der physischen Umwelt verstanden werden, nachdem eine bewußte Auseinandersetzung mit dem Gegenstand der Veränderung stattgefunden hat. Insofern ist die früher als Soziotechnik abgetane Akzeptanzforschung nicht darauf angelegt, durch psychologische Tricks die Bereitschaft zur Hinnahme von neuen Technologien oder anderer Maßnah-

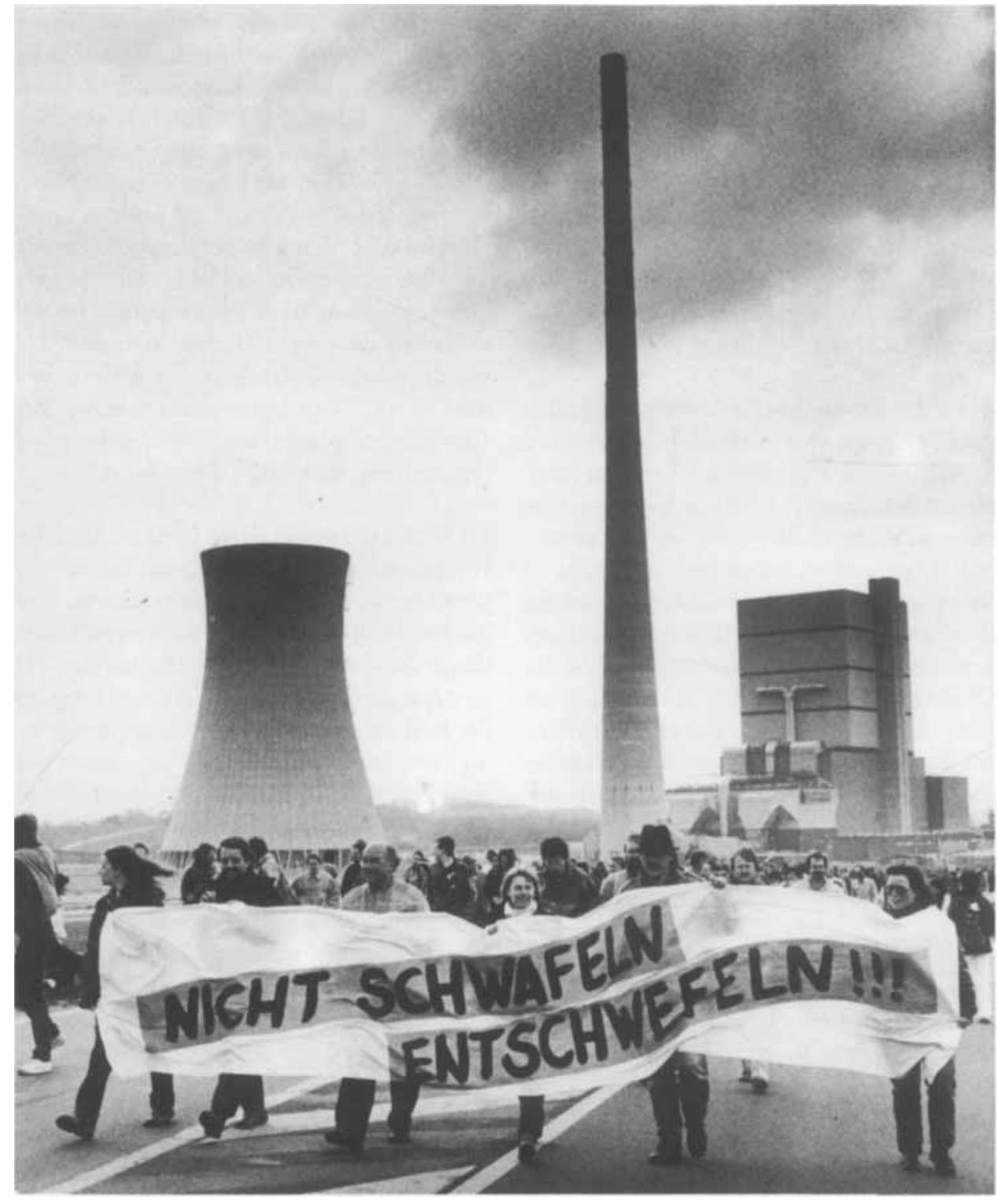

men zu fördern, sondern den Wahrnehmungsund Bewertungsprozeß von Individuen und sozialen Gruppen nachzuzeichnen und zu analysieren.

Die folgenden Ausführungen haben das Ziel, die bisherigen Ergebnisse der Akzeptanzforschung im Bereich neuer Technologien in gestraffter Form vorzustellen und zu diskutieren. Wenn auch in diesem Bericht im wesentlichen nur die Ergebnisse der vielfältigen Studien zu diesem Thema angeschnitten werden können, so sollen doch an einigen Stellen auch die Methoden und Verfahren beschrieben werden, die zu den Ergebnissen geführt haben. Dabei geht es vor allem darum zu zeigen, daß mit Techniken der empirischen Sozialforschung in Analogie zur naturwissenschaftlichen Forschungspraxis relativ zuverlässige Aussagen über das Phänomen der Technikakzeptanz gewonnen werden können.
Die unterschiedlichen Arten von Technikakzeptanz

In den westlichen Industriegesellschaften gibt es keine durchgängige Technikfeindlichkeit, sondern lediglich ein zunehmendes Erkennen der Ambivalenz technologie-induzierter Folgen. Innerhalb der westdeutschen Bevölkerung findet man unterschiedliche Strömungen und unterschiedliche Technikbilder. Junge Menschen urteilen meistens etwas skeptischer über Technik als ältere, Menschen im Berufsleben sind weniger von den negativen Auswirkungen der Technik überzeugt als Menschen außerhalb des Berufslebens, und Personen mit wirtschaftsfremden Tätigkeiten lehnen moderne Technologien eher ab als Personen, die in Industrie und Wirtschaft arbeiten. Wenn man überhaupt von einer allgemeinen Tendenz reden kann, so können wir aus den vielfältigen Umfragen der letzten Jahre 
nur die Schlußfolgerung ziehen, daß die Ambivalenz der Technik stärker wahrgenommen wird als früher. Nach wie vor ist die überwiegende Mehrheit davon überzeugt, daß Technik zum Segen der Menschheit beiträgt, aber eben auch negative Konsequenzen hat. Versucht man die Frage der Technikakzeptanz weiter zu differenzieren, so ist es notwendig, von unterschiedlichen Akzeptanzbereichen auszugehen. In der Akzeptanzforschung unterscheiden wir vier Funktionsmuster:

- die konsumtive Akzeptanz: Damit ist die Aufnahme derjenigen Technologien gemeint, die als technische Geräte im privaten Haushalt und zur privaten Lebensführung eingesetzt werden. In diesem Bereich gibt es so gut wie keine Akzeptanzprobleme. Hifi-Geräte, Motorräder, Küchengeräte, Heimbohrer und anderes mehr sind genauso beliebt wie eh und je. Selbst moderne Konsumtechnologien, wie der Kompakt-Disk-Player, Telespiele und sogar der Heimcomputer, stoßen keineswegs auf prinzipielle Ablehnung. Akzeptanzprobleme gibt es allenfalls bei teilöffentlichen Gütern, die auf staatlichen Vorleistungen beruhen. Wenn beispielsweise neue Kommunikationstechnologien auf staatlichen Infrastrukturleistungen, wie der Verkabelung, aufbauen, deren Kosten von der Gesamtheit übernommen werden müssen, sind Proteste eher wahrscheinlich.

- Technologieakzeptanz am Arbeitsplatz: Dieser Begriff umfaßt die Technologien, die am Arbeitsplatz zur Herstellung von Produkten oder Dienstleistungen im kommerziellen Rahmen benutzt werden. Auch in diesem Bereich stellt man nur in wenigen Wirtschaftsbereichen massive Widerstände gegen neue Technologien fest, während in den meisten Fällen Technologieproteste ausblieben. Vor allem im Bereich der Informationstechnologien läßt sich nachweisen, daß Personen nach einer gewissen Eingewöhnungszeit gegenüber diesen Innovationen wesentlich positiver eingestellt waren als der Bevölkerungsdurchschnitt.

Aus einer Reihe von internationalen Vergleichsstudien ist bekannt, daß die deutsche Wirtschaft häufig nicht die Vorreiterrolle in der Innovationsgeschwindigkeit übernimmt, sondern neue Technologien eher behutsam einführt. Die behutsame Einführung bringt es mit sich, daß die Innovationen von der Belegschaft eher akzeptiert werden und die neuen Technologien auch intensiver genutzt werden als in Vergleichsländern, in denen der Umbruch radikaler vorgenommen wurde.
- Die Akzeptanz als Nachbar: Mit diesem Begriff wird die Aufnahme derjenigen Technologien umschrieben, denen der Mensch als Anwohner "ungefragt" ausgesetzt wird. Vor allem in diesem Bereich haben sich die hinlänglich bekannten Akzeptanzprobleme entzündet. Kernkraftwerke, große Industrieansiedlungen oder Sondermülldeponien sind zunehmend in Legitimationsschwierigkeiten geraten. Dabei tritt häufig eine Dichotomie zwischen Produkt und Produktion zutage. Nach Umfragen des Instituts für Demoskopie in Allensbach gibt es kaum eine Energieform, die mehr geschätzt wird, als der elektrische Strom, und gleichzeitig kaum eine Anlage, die insgesamt negativer eingeschätzt wird, als Kraftwerke. Der Düsseldorfer Sozialpsychologe Hans-Christian Röglin hat diese Tatsache auf eine einfache Formel gebracht: „Wir lieben die Produkte der Industriegesellschaft, aber wir hassen die Art, wie sie hergestellt werden." Angelpunkte der Kritik an Großtechnologien sind zum einen die fehlende Möglichkeit der Anwohner, an der Gestaltung ihrer physischen Umgebung maßgeblichen Anteil zu nehmen, und zum anderen das Mißtrauen gegenüber der Behauptung von Betreibern und Genehmigungsbehörden, daß von derartigen Anlagen auch wirklich keine Gefährdung ausgehe.

- Symbolakzeptanz: Unter diesem Begriff wird die Verkettung von symbolischen Attributen mit bestimmten Technologien verstanden. Bestimmte Technologien erhalten dabei Symbolcharakter in der gesellschaftlichen Auseinandersetzung um die kollektive Verbindlichkeit von Wertorientierungen und politischen Zielvorstellungen. Häufig treten dabei die instrumentellen Vor- und Nachteile einzelner Technologien zugunsten der ihnen zugeschriebenen Stellvertreterrolle für wettstreitende Zukunftsentwürfe in den Hintergrund.

Wenn wir also von Akzeptanzkrise oder Akzeptanzverweigerung reden, ist es notwendig, zwischen den verschiedenen Ebenen der Akzeptanz zu differenzieren. Von Akzeptanzverweigerung kann allenfalls im Bereich der Nachbarschafts-Akzeptanz geredet werden.

\section{Psychologische Faktoren der Technikwahrnehmung}

Unter psychologischen Gesichtspunkten gibt es sechs zentrale Wahrnehmungsmuster, die das Akzeptanzverhalten von Menschen weitgehend steuern:
- Die Angst vor dem Verlust zukünftiger Handlungsmöglichkeiten: Sofern Personen davon überzeugt sind, daß ihre eigene oder die Handlungsfreiheit der anderen in Zukunft eingeschränkt werden könnte, entwickeln sie in der Regel alternative Handlungsstrategien oder versuchen auf andere derart einzuwirken, daß veränderte Optionen der Zielerreichung angestrebt werden.

Das Streben nach Sicherung von Handlungsfreiheit läßt sich anhand eines sozialpsychologischen Experiments, das die sozialwissenschaftliche Abteilung der Programmgruppe "Technik und Gesellschaft" der Kernforschungsanlage Jülich (KfA) im Jahre 1979 durchgeführt hat, anschaulich illustrieren. Zwei zufällig ausgesuchte Gruppen von Versuchspersonen wurden vom Versuchsleiter gebeten, an einem pharmazeutischen Erprobungstest teilzunehmen. Angeblich ging es darum, drei verschiedene Kapselumhüllungen auf unangenehme Nebenwirkungen zu testen. Nach den Worten des Versuchsleiters enthielt die erste Kapsel eine radioaktive Ummantelung, die zweite eine bakterielle Umhüllung und die dritte einen Säuremantel, wobei sich alle drei Kapseln schneller im Magen auflösen als herkömmliche Materialien. Ein gesundheitliches Risiko sei bei allen drei Kapseln nicht gegeben. In Wirklichkeit handelte es sich bei allen drei Kapseln um absolut identische, handelsübliche Vitamintabletten. Die Mitglieder der ersten Gruppe durften sich eine Kapsel aussuchen, die zweite Gruppe erhielt vom Versuchsleiter jeweils eine Kapsel zugewiesen. Nach der Einnahme der Kapsel mußten die Versuchspersonen einen Fragebogen ausfüllen, in dem sie über eventuelle Beschwerden (Magendrücken, Unwohlsein usw.) Auskunft geben sollten.

Das Ergebnis dieses Versuches ist in Abbildung 1 dargestellt. Obgleich alle Versuchspersonen identische Kapseln geschluckt hatten, gaben die Versuchspersonen der Gruppe 2, die keine Auswahl hatten treffen dürfen, im Schnitt doppelt so oft an, sie fühlten sich unwohl, als die Personen, die eine Kapsel aussuchen durften. Dieses Ergebnis war völlig unabhängig davon, welche Kapselummantelung im einzelnen gewählt oder aufgezwungen wurde. Interessant am Rande ist auch die Tatsache, daß die angeblich radioaktiven Kapseln bei beiden Gruppen am häufigsten Beschwerden hervorriefen.

Daß Freiwilligkeit eine wesentliche Größe bei der Risikowahrnehmung darstellt, ist seit lan- 
gem wichtiger Bestandteil der psychologischen Risiko- und Entscheidungstheorie. Allerdings wurde erst mit diesem Kapselexperiment diese Beziehung empirisch eindeutig nachgewiesen.

- Die Sicherstellung der Beherrschbarkeit von Technologien: So sehr Technik als menschliches Artefakt für das gesellschaftliche Ưberleben und das soziale Handeln notwendig ist, so sehr fürchten sich die Menschen davor, durch ihre eigenen Instrumente in ihrem Handeln und Verhalten eingeschränkt und reglementiert $\mathrm{zu}$ werden. Ausdrücklich muß betont werden, daß es nicht die Undurchschaubarkeit von Technologien ist, die Angst einflößt, sondern die Vorstellung, nicht mehr Herr über die eigene technologische Entwicklung zu sein. So gehen beispielsweise von Hifi-Anlagen oder mikroprozessor-gesteuerten Waschmaschinen keine angstauslösenden Impulse aus, weil die Benutzer die völlige Kontrolle über den „Ein- und Ausschalter" sowie die Programmwahl haben. Die Tatsache, daß nicht die Undurchschaubarkeit, sondern die Beherrschbarkeit zentraler Angelpunkt der Technikakzeptanz ist, wird von Vertretern sogenannter „sanfter" Technologien häufig übersehen.

- Bedrohung von Privatheit: Alle Technologien, die als Eingriff in das eigene Privatleben wahrgenommen werden, stoßen schnell auf Akzeptanzgrenzen. Dies gilt sowohl für Informationstechnologien, als auch für Großtechnologien, bei denen weitreichende staatliche Kontrollen notwendig sind. In einer Welt, in der zunehmend soziale Beziehungen anonymisiert und Inhalte der Lebenswelt professionalisiert werden, reagiert der Mensch sensibel auf die Bedrohung der Privatheit.

- Bedrohung der Arbeitstätigkeit: Menschen empfinden Technologien als eine Bedrohung, sofern durch ihren Einsatz das Prestige ihres eigenen Arbeitseinsatzes sinkt oder sie sogar ihren Arbeitsplatz verlieren können. Technologien am Arbeitsplatz werden um so mehr geschätzt, je mehr sie das eigene Prestige innerhalb des sozialen Umfeldes erhöhen und je mehr monotone und eintönige Tätigkeiten substituiert werden (aber nicht unbedingt manuelle!). Aus empirischen Untersuchungen wissen wir, daß Innovationsschübe in Betrieben meistens gleichzeitig mit Dequalifikationen und Höherqualifikationen einhergehen. Insofern ist es immer erforderlich, durch begleitende Maßnahmen Defizite auszugleichen.

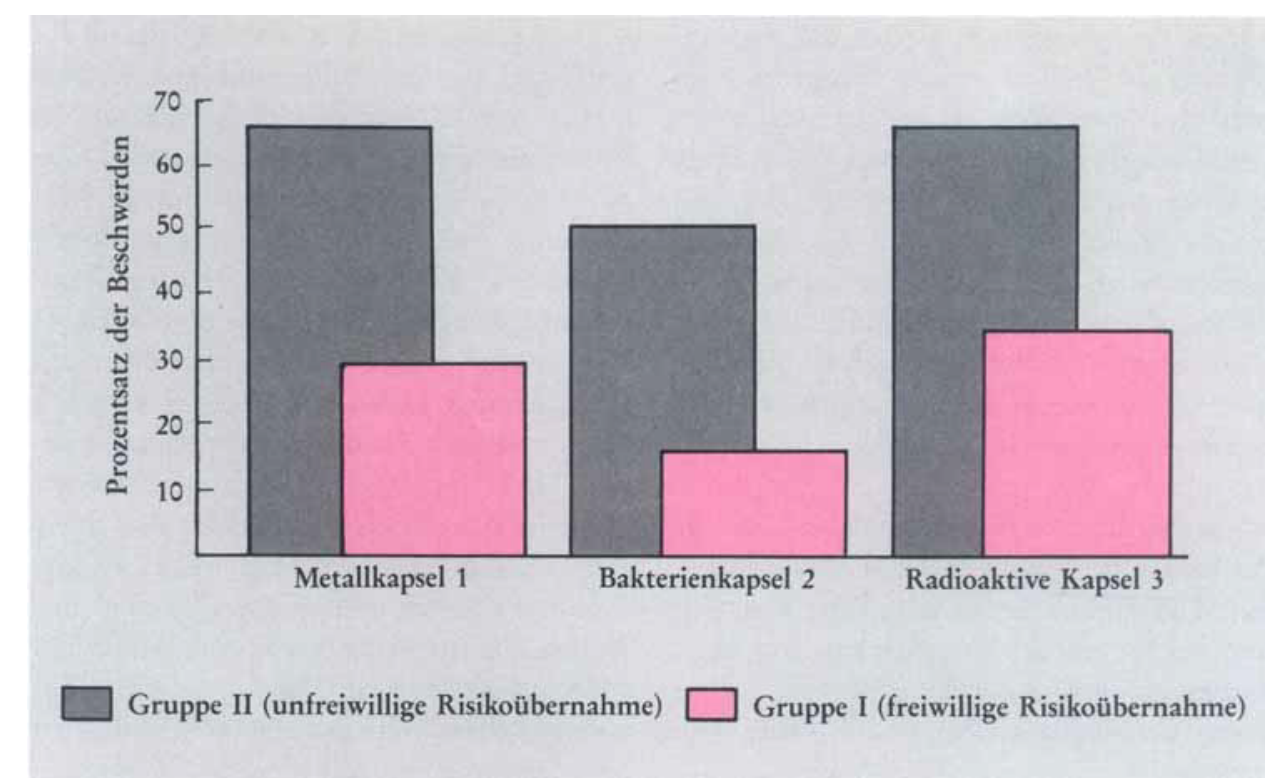

- Verlust von Vertrauen in die Träger von Innovationen: In immer stärkerem Maße ist der Mensch der modernen Industriegesellschaft auf vermittelte Informationen angewiesen. Während früher der Anteil der direkt wahrnehmbaren Erfahrung rund 40 bis 60 Prozent des gesamten gespeicherten Wissens aus machte, besteht unser Wissen heute zu über 90 Prozent aus vermittelten Informationen. $\mathrm{Ob}$ es in Nicaragua, China, Afghanistan oder Neuseeland wirklich so aussieht, wie uns die Fernsehkorrespondenten berichten, entzieht sich bei der überwiegenden Mehrheit der Bevölkerung der persönlichen Erfahrungswelt.

Das gleiche gilt auch für die Vermittlung technologischer Informationen. Je geringer aber die Möglichkeiten sind, durch eigene Erfahrung Informationen zu überprüfen, desto größer ist der Anreiz für Informanten zur Manipulation von Meinungen und desto leichter ist es, widersprechende Meinungen in technologische Debatten einzubringen, weil man sie vor der Öffentlichkeit nicht nachzuweisen braucht (und auch nicht nachweisen kann). Aus dem gleichen Grund reagieren die Stellvertreter der öffentlichen Meinung (vor allem die Medien) auf echte oder vermeintliche Fehlinformationen und Informationsdefizite besonders empfindlich. Nicht zuletzt das Spannungsverhältnis zwischen unabdingbarem Vertrauensvorschuß und faktischem Vertrauensentzug hat die Dramaturgie bei den Nachbarschaftsakzeptanz-Konflikten geprägt. Denn als Anrainer von technischen Anlagen ist man in besonderem Maße auf sachgerechte und ehrliche Informationen der Betreiber und Genehmigungsbehörden angewiesen. Da die meisten Personen die Richtigkeit der sich häu-
Abb. 1. Ergebnisse des Kapsel-Experiments (Placebo-Effekt). Trotz identischer Kapseln empfanden mehr als doppelt so viele Versuchspersonen subjektive Beschwerden, wenn sie eine Kapsel zugewiesen bekamen, im Vergleich zu der Experimentanordnung, bei der sie eine aus drei Kapseln auswählen konnten.

fig widersprechenden Informationen nicht überprüfen können, gewinnt die Ausstrahlung von Glaubwürdigkeit besonderes Gewicht. Nicht mehr der Inhalt ist der Maßstab für kognitive Urteilsbildung, sondern die Wahrnehmung der sozialen Situation bei der Informationsübertragung und die Einschätzung der Informationsquelle.

Um die Glaubwürdigkeit verschiedener Akteure im Konflikt um die Kernenergie zu messen, wurden bei einer Befragung durch die Programmgruppe "Technik und Gesellschaft" der KfA Jülich nach dem Zufallsverfahren ausgewählte Bürger gebeten, auf einer Skala von 1 bis 4 die Glaubwürdigkeit von 20 verschiedenen Instituten und Personengruppen abzuschätzen ( 1 - sehr glaubwürdig, 2 = eher glaubwürdig, 3 = eher nicht glaubwürdig, 4 = gar nicht glaubwürdig). Vorgegeben wurde eine fiktive Situation, in der die 20 Institutionen im Fernsehen zum Thema Kernenergie zu Wort kamen.

Die Ergebnisse sind in Tabelle 1 wiedergegeben. Die Tabelle gibt über eine Reihe von Sachverhalten Auskunft:

- Wenn jemand Glaubwürdigkeit in Fragen 
Tabelle 1. Verschiedenen Institutionen zugeschriebene Glaubwürdigkeit in Fragen der Kernenergie (Mediane; Spannweiten:

1 = sehr glaubwürdig, 4 = gar nicht glaubwürdig).

\section{Institution}

Gesamt-

Mittelwert

t-Test (p)

\section{Professor an einem}

Kernforschungszentrum

Wissenschaftler/Universität

Reaktorsicherheitskommission

Bundesminister BMFT

Wissenschaftler einer

Bürgerinitiative

Vertreter der Ärzteschaft

Bundeskanzler

Sprecher einer Bürgerinitiative

Aufsichtsratsvorsitzender

Fernsehkommentator

SPD-Sprecher

CDU-Sprecher

Journalist

Politiker

FDP-Sprecher

Manager eines Industriebetriebs

Lokalpolitiker

Gewerkschaftsvorsitzender

Pfarrer

Bundesvorsitzender des DFB

Durchschnittswert

$\begin{array}{rrr}2,71 & 2,80 & 2,64 \\ +0,23 & +0,64 & +0,38\end{array}$

der Kernenergie zugesprochen wird, dann sind es am ehesten Vertreter von Wissenschaft und Technik oder von fachgebundenen politischen Instanzen (beispielsweise vom Bundesministerium für Forschung und Technologie).

- In der Frage der Vergabe von Glaubwürdigkeit gegenüber wissenschaftlichen Institutionen gibt es nur geringe Unterschiede zwischen Gegnern und Befürwortern der Kernenergie. Beide Gruppen vertrauen diesen Institutionen am meisten.

- Politisch zentrale und wirtschaftlich einschlägige Institutionen belegen überwiegend mittlere Skalenplätze, während allgemeine gesellschaftliche Institutionen, wie Gewerkschaften und Kirchen, sowie unspezifische Sozialpositionen, wie Manager oder Lokalpolitiker, das untere Ende der Skala bestimmen. minativ für Position und Einstellung. beiden Fällen werden diffuse Vorstellungen und Ängste auf ein Objekt hin kanalisiert, das stellvertretend für positive oder negative Emotionenbündel eine Manifestation der eigenen Bewunderung oder Verachtung bildet. In psychologischer Hinsicht handelt es sich dabei um eine symbolische Befreiung von psychischem Druck (Kathar), indem man die häufig unbewältigten eigenen Emotionen aus dem eigenen Körper auf ein fremdes Objekt überträgt.

Wie sehr einzelne Technologien mit sozialen und politischen Symbolen assoziiert werden, läßt sich am Beispiel der Kernenergie auch empirisch nachweisen. Bei einer Risikoakzeptanz-Studie der Programmgruppe ,Technik und Gesellschaft" der KfA Jülich wurde der Einfluß allgemeiner soziopolitischer Einstellungen für die Risiko-Nutzen-Abwägung bei der Kernenergie in der Bevölkerung untersucht. Die Befragten wurden zunächst gebeten, das Verhältnis von Nutzen und Risiko der Kernenergie auf einer Skala von -3 bis +3 einzuordnen und anschließend Fragebatterien zu allgemeinen sozialen und politischen Themen zu beantworten. Diese Fragebatterien umfaßten fünf Einstellungsmuster:

- das Vertrauensverhältnis zu Wissenschaft und Technik,

- die Präferenz für konservativen Lebensstil,

- der Wunsch nach Partizipation,

- die Neigung zur politischen Apathie,

Wie sind diese Daten zu interpretieren? Alle Befragten sind sich darüber einig, daß Aussagen zur Kernenergie von fachgebundenen Institutionen am glaubwürdigsten sind. Politische und gesellschaftliche Institutionen werden - je weniger sie mit Sachkompetenz assoziiert werden können - in der Glaubwürdigkeit auch um so schwächer eingestuft. Je mehr wissenschaftliche Institutionen positionsgebundene Haltungen einnehmen, wie Bürgerinitiativen oder die Reaktorsicherheitskommission, desto stärker wirken sie diskri-

Symbolfunktionen von Technologien: Auf die besondere Bedeutung von Technologien als Symbole für gesellschaftspolitische Auseinandersetzungen ist bereits im ersten Punkt hingewiesen worden. Darüber hinaus können Technologien auch die Rolle des Sündenbocks oder von Identifikationsobjekten spielen. In
- das Bewußtsein über die Notwendigkeit von Umweltschutz.

Die Ergebnisse der Beziehungen zwischen Einstellungsmuster und Risiko-Nutzen-Abschätzung der Kernenergie sind in Abbildung 2 wiedergegeben. Ein geringes Vertrauen in die Aussagen von Wissenschaftlern und Technikern verbunden mit einer hohen Priorität für Umweltschutz bedingt eine a priori negativere Sichtweise des Risikos „Kernenergie". Umgekehrt ist Vertrauen in Wissenschaft und Technik und ein geringes Niveau von Umweltschutz-Bewußtsein eine Ausgangshaltung, die eher positive Assoziationen zur Kernenergie begünstigt. Auch ein konservativer Lebensstil ist heute eher eine Voraussetzung für eine befürwortende Haltung gegenüber der Kernenergie geworden. Dagegen spielt die Frage der politischen Grundeinstellung „Aktivismus versus Fatalismus“ nur eine 
2

geringfügige Rolle. Dies wird durch die Tatsache bestätigt daß von allen Bevölkerungsgruppen und in allen politischen Lagern Kernenergie mit relativ ähnlichem Symbolgehalt versehen wird.

Die Akzeptanzforschung hat für alle diese sensitiven Bereiche empirische und theoretische Studien vorgelegt. Bislang stand dabei die Frage im Vordergrund, warum es bei manchen Technologien Akzeptanzprobleme gibt und bei anderen nicht. Mit Hilfe der hier nur in Ansätzen beschriebenen Systematik dürfte es in Zukunft aber auch möglich sein, bei neu auftretenden Technologien Akzeptanzprobleme im voraus zu diagnostizieren und entsprechende Gestaltungsvorschläge für die Einführung neuer Technologien zu erarbeiten. Um Mißverständnisse $\mathrm{zu}$ vermeiden, muß deutlich gesagt werden, daß die hier aufgeführten Akzeptanzmechanismen viel zu fundamental und zentral im menschlichen Bewußtsein angelegt sind, als daß sie durch Propaganda, Public Relations oder andere Mittel der Öffentlichkeitsarbeit quasi manipulativ überwunden werden könnten. Vielmehr muß der Handlungsspielraum genutzt werden, der durch die Formbarkeit der Technologien für verschiedene $Z$ wecke und deren organisatorisches Umfeld vorgegeben ist.

\section{Technikakzeptanz im historischen Kontext}

Das Unbehagen an der Modernität ist keine moderne Erscheinung. Seit Beginn der Industrialisierung sind Technologie und Industrialisierung ins Kreuzfeuer der Kritik geraten. Von der Romantik, über den Maschinensturm bis zur Jugend- und Heimatschutzbewegung der zwanziger Jahre zieht sich eine deutliche Linie der Technikkritik, die Ende der sechziger Jahre von den Umweltgruppen wieder aufgenommen wurde. Die Protestbereitschaft darf jedoch nicht als eine kontinuierliche Bewegung verstanden werden, sondern vielmehr als eine zyklische, immer wieder neu aufflammende und sich selten auf historische Vorbilder berufende Spontanaktion von Bürgern. Seit Beginn der Industrialisierung ist dieses latente Protestpotential gegen „technische Rationalität", „soziale und ökonomische Differenzierung" und „zunehmende Industrialisierung" vorhanden, das immer dann an politischer Kraft und sozialem Einfluß gewinnt, wenn die Industriekultur selbst krisenhafte Erscheinungen hervorbringt und gleichzeitig mehr und mehr Menschen unter den Auswirkungen dieser wirtschaftlichen Erscheinun-

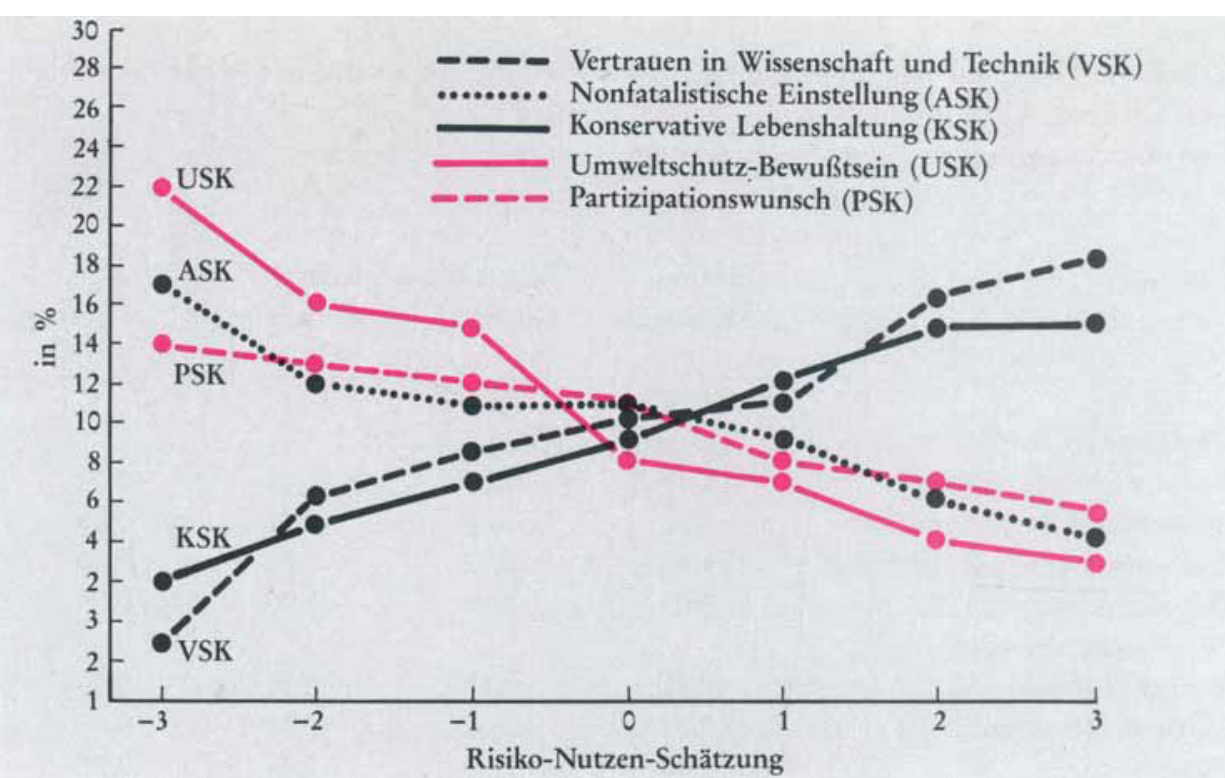

gen zu leiden haben (Umweltverschmutzung, Arbeitslosigkeit, Entfremdung von Natur und Produkt). Die Träger des latenten Protestpotentials gegen die Industriegesellschaft sind sich einig in der Ablehnung eines durchrationalisierten Wirtschaftssystems und eines auf Zweckrationalität beruhenden Verwaltungssystems. Politisch wirksam wird dieses Potential aber erst dann, wenn es im Verlauf ökonomischer Zyklen einerseits zu strukturellen Krisenerscheinungen kommt (Arbeitslosigkeit, Innovationslähmung, ungleichgewichtige Wirtschaftsentwicklung) und andererseits diese Defizite in der Bevölkerung als „Fehler“ des Steuerungssystems wahrgenommen werden.

Offenkundig empfinden auch zum jetzigen Zeitpunkt viele Menschen die Probleme von Industrialisierung und Technik als so schwerwiegend, daß die herkömmlichen Leitbilder des gesellschaftlichen Handelns für die Orientierung im Alltag nicht mehr ausreichen. Sie vertrauen auch nicht mehr auf die Institutionen und Verfassungsorgane, die bei gesellschaftlichen Problemen Lösungen erarbeiten müssen. Schließlich empfinden sie ein Unbehagen an dem System politischer und wirtschaftlicher Entscheidungsfindung.

Lassen sich für diesen Sinneswandel Gründe angeben? Ohne eine erschöpfende Liste der Motive aufstellen zu wollen, erscheinen mir folgende sieben Punkte besonders erwähnenswert:

- Durch verlangsamtes wirtschaftliches Wachstum, verbunden mit einem relativ hohen Lohnniveau, ist die Zahl der durch Ratio-
Abb. 2. Verschiedene politisch-soziale Einstellungen in Abhängigkeit von der RisikoNutzen-Abschätzung der Kernenergie (Mittelwerte der jeweils standardisierten Skala). Je größer das Vertrauen in die Leistungsfähigkeit von Wissenschaft und Technik (VSK) und je geringer die Sensibilität für Umwelt und Natur (USK), desto eher ist eine positive Risiko-Nutzen-Schätzung der Kernenergie zu erwarten.

nalisierung freigesetzten Arbeitskräfte größer als die Zahl der neugeschaffenen Arbeitsplätze. Neuinvestitionen sind gegenüber Ersatzinvestitionen in den Hintergrund getreten. Mit diesem strukturellen Wandel ändert sich auch die Einstellung der Bevölkerung zum technischen Fortschritt. Wurde er zunächst als Form der Humanisierung des Arbeitslebens empfunden, so wird er heute zunehmend als Bedrohung für die Sicherheit des Arbeitsplatzes angesehen.

- Zusammen mit der Angst vor neuen Technologien als "Jobkiller" wächst die Entfremdung des Menschen gegenüber technischen Projekten in seiner physischen Umgebung. Die Größe und Komplexität moderner technischer Anlagen machen die Umwelt für den Betrachter unüberschaubar und im Gefahrenpotential bedrohlich. Auch die sozialen Konsequenzen sind häufig schwer verkraftbar. Es kommt zu einer manifesten Auflehnung gegen technische Projekte, wie man sie bei Demonstrationen gegen Atomkraftwerke erlebt hat.

- Steht in der Aufbauphase eines Landes (wie in der Bundesrepublik Deutschland nach dem 
2. Weltkrieg) das quantitative Wachstum an erster Stelle der politischen Prioritätenliste, so treten nach Ablauf von Sättigungsprozessen und ausreichender Konsumausstattung qualitative Bedürfnisse in den Vordergrund (saubere Umwelt, Freizeit, Selbstverwirklichung), die aus prinzipiellen wie organisatorischen Gründen nur teilweise vom bestehenden Wirtschafts- und Sozialsystem befriedigt werden können. Vor allem aber sind qualitative Bedürfnisse kaum mehr gesamtgesellschaftlich konsensfähig. Jede Gruppe der Gesellschaft stellt sich beispielsweise unter „Lebensqualität" etwas anderes vor. Politische Zielvorstellungen werden dann schnell zu Etiketten, die beliebig gefüllt werden können. Das System vermochte also in der Vergangenheit dem ursprünglichen Leitbild nach Bedürfnisbefriedigung gerecht zu werden, kann sich aber bei gegenwärtig veränderter Bedürfnisstruktur dem eigenen Leitbild nach Ermöglichung individueller Entfaltungschancen immer weniger anpassen.

- Erschüttert hat den Glauben an das eigene Leitbild auch die Erkenntnis der Grenzen des Wachstums und der zunehmenden Umweltbelastung. Die Hoffnung auf eine dauerhafte Expansion des notwendigerweise dynamischen Produktionssystems wurde gebrochen - eine Umorientierung erscheint ,irgendwann" unvermeidlich.

- Eng verknüpft mit der Belastung der Umwelt sind zunehmende Einsicht in und Betroffenheit über die sozialen Kosten wirtschaftlicher Produktion. Je mehr Elemente der Lebenswelt vom ökonomischen System aufgrund der Kommerzialisierung des Alltags beeinflußt werden und je mehr sich das ökonomische System aufgrund immanenter Wachstumstendenzen ausdehnt, desto mehr weitet sich die Kluft zwischen betrieblicher Rentabilität und sozialem Nutzen. Dies führt einerseits im Bewußtsein großer Bevölkerungsteile zu einer negativen Einstellung der Privatwirtschaft gegenüber, veranlaßt andererseits den Staat zu stärkeren Eingriffen in das wirtschaftliche Geschehen.

- Die Ende der sechziger Jahre aufkommenden euphorischen Erwartungen an einen verstärkten Eingriff des Staates durch Globalsteuerung und Bereitstellung öffentlicher Güter gingen bald (besonders in USA) in herbe Enttäuschung über: Die hinzugewonnene Macht wurde teilweise von der Exekutive extensiv genutzt, so daß in der Öffentlichkeit der Eindruck des Machtmißbrauchs entstand.
Das private Wirtschaftsgeschehen, das zwar als ungerecht, aber immerhin als flexibel und effizient empfunden wurde, wurde durch Bürokratie und ein Übermaß an Verordnungsvorschriften überwuchert. Die zwangsläufige Anonymisierung des staatlichen Leistungsangebots vertiefte das Gefühl der Entfremdung. Die Zentralisierung der Entscheidungsgewalt überging die speziellen Anforderungen lokal gewachsener Strukturen.

- Große Teile der Bevölkerung wollen und können sich nicht damit abfinden, selbst im Überfluß zu leben und gleichzeitig zu wissen, daß Millionen Menschen hungern und ihre einfachsten Bedürfnisse nicht befriedigen können. Die offensichtlichen Mißerfolge der bisherigen Entwicklungspolitik haben sie dazu veranlaßt, über die Forderung nach Veränderungen der Gesellschaftsstruktur eine neue globale Ausgangslage herbeizuführen, die einen wirkungsvollen Ausgleich zwischen Industrieländern und Entwicklungsländern ermöglichen soll.

Über die strukturellen Probleme des Wirtschaftssystems und seine zyklischen Krisen hinaus befindet sich auch das politische System unter öffentlichem Druck. Die Distanz zwischen der Regierung und den sie tragenden Parteien, ein durch Komplexität der Entscheidungen hervorgerufener Funktionsverlust der Parlamente, die Vertiefung des öffentlichen Mißtrauens gegen angeblich neutrale Sachverständige oder gegen scheinbare Sachentscheidungen, die sich im nachhinein allzuoft als Interessendurchsetzungen entpuppten, durch Interessengruppen beherrschte Kanäle der politischen Einflußnahme, die Aufdeckung von echten und vermeintlichen Übergriffen der Staatsgewalt auf die Freiheit einzelner Bürger sowie das Nichtreagieren auf neue Fragestellungen, die von gesellschaftlichen Gruppen an das politische System herangetragen werden, sind wohl die wichtigsten Symptome einer zunehmenden Entfremdung zwischen Staatsgewalt und Staatsbürger. Die Aufzählung ließe sich sicher noch fortsetzen.

Das zyklische Auftreten von Protestbewegungen hat in der Vergangenheit selten zu starken Kursänderungen der offiziellen Politik geführt. Entweder wurden Teile der Inhalte langsam in die offizielle Politik integriert oder aber von Subgruppen in der Gesellschaft bis zu ihrer Bestandsauflösung weitergelebt.

Inwieweit die neuen politischen Bewegungen, die sich für mehr Natur und weniger Technik einsetzen, in Zukunft fortbestehen können, läßt sich zur Zeit schwer prognostizieren. Wie alle sozialen Bewegungen sind auch sie dem Zeitreiheneffekt für Aufmerksamkeit (issueattention cycle) ausgesetzt, der allen sozialen Themen nur eine bestimmte Lebensdauer im allgemeinen öffentlichen Interesse einräumt. Gleichzeitig ist aber davon auszugehen, $\mathrm{daß}$ in der Bevölkerung die Resonanz auf das neue Thema „Umweltverträglichkeit" durchgängig positiv ist, so daß „Grüne Weltbilder" auch in bürgerlichen Kreisen salonfähig geworden sind.

\section{Wertwandel und Technikakzeptanz}

Wenn auch Protest und Opposition gegen Technik und Industrialisierung weitgehend auf Randgruppen der Gesellschaft beschränkt sind, so zeichnet sich dennoch in der breiten Bevölkerung eine Veränderung dominanter Werthaltungen ab. Im Gegensatz zur populären Vorstellung, daß die in den fünfziger und sechziger Jahren dominierenden materiellen Werte durch post-materielle abgelöst würden, erkennen wir aus der Summe der empirischen Studien, daß die breite Mehrheit der Bevölkerung eine inhomogene Mischung von leistungsbezogenen, konsumbezogenen, naturbezogenen und lebensqualität-bezogenen Werten entwickelt hat. In einer jüngst abgeschlossenen Studie der Programmgruppe "Technik und Gesellschaft" der KfA Jülich zum Thema ,Sozialverträglichkeit von Energieversorgungssystemen“ wurden zufällig ausgewählte Bürger gebeten, die Wichtigkeit von Kriterien, mit denen man Energiesysteme beurteilen könne, abzuschätzen. Das Kriterienraster stellt nichts anderes als eine umfassende, thematisch geordnete Liste relevanter Wertvorstellungen im Hinblick auf Energiesysteme dar. Personen unterscheiden sich zunächst dadurch, daß sie die einzelnen Werte für unterschiedlich wichtig halten. Um die Wertpräferenzen der befragten Bürger zu erfassen, wurden sie gebeten, den acht Hauptkriterien (und damit den durch sie repräsentierten Werten) Gewichte zuzuordnen, deren Summe jeweils 100 ergeben mußte. Abbildung 3 zeigt, wie die Gewichte im Mittel verteilt wurden. Die Kriterien mit den im Schnitt höchsten Gewichten sind demnach „Gesundheit und Sicherheit", „Umweltauswirkungen“" und „Versorgungssicherheit". Eine Mittelstellung nehmen die Kriterien „Volkswirtschaftliche Auswirkungen", „Finanzielle und materielle Auswirkungen" und „Soziale Auswirkungen" ein. Die als am wenigsten wichtig für die Beurteilung von Energiesystemen ein- 
geschätzten Kriterien sind „Politische Auswirkungen" und "Internationale Auswirkungen".

Bei der konkreten Anwendung dieser Kriterien zeigte sich bei den meisten Bürgern eine deutliche Ambivalenz im Zielkonflikt zwischen Umwelt und Wirtschaftlichkeit. Wenn es auch kleinere Gruppen gab, die eindeutig dem einen oder anderen Pol Priorität einräumten, so war die überwiegende Mehrheit zwischen beiden Zielvorstellungen hin und her gerissen. Aus diesem Resultat läßt sich die Forderung an die Politik ableiten, bei allen technischen Lösungen auf Wirtschaftlichkeit und Umweltverträglichkeit gleichzeitig Wert zu legen.

Der Berliner Sozialökonom Burkhard Strümpel hat diese Ambivalenz einmal als das klassische „Gefangenen-Dilemma" bezeichnet, da für die einzelne Person jede Handlungsalternative mit Wertverletzungen verbunden ist. Als Folge dieses "Uneins-Sein“ mit sich selbst lassen sich Resignation, virulentes Aufbäumen gegen offizielle Entscheidungsträger oder Rückzug aus der komplexen Welt in rigorose Weltbilder (bis hin zu Sekten) beobachten. In fast allen Fällen führt aber der Wunsch nach Auflösung der inneren Widersprüche zu einer stark selektiven Aufnahme von Informationen und einer häufig sehr pauschalen Etikettierung von Informationsträgern nach vorgefaßten Meinungsbildern. Einige Mechanismen, wie diese Reduktion der komplexen Wirklichkeit zu einem einfacheren Weltbild vonstatten geht, möchte ich an dieser Stelle kurz ausführen:

- Die Verteilung von Glaubwürdigkeit nach vermuteter Interessenlage: Wegen des Übermaßes an vermitteltem Wissen und der Unmöglichkeit, Informationen auf ihren Wahrheitsgehalt $z u$ überprüfen, müssen Individuen eigene Selektionskriterien entwickeln, um bei sich widersprechenden Informationen der einen oder anderen Quelle Glaubwürdigkeit zuoder abzusprechen. Seit Mitte der sechziger Jahre können wir beobachten, daß die Glaubwürdigkeit zunehmend von der vermuteten Interessengebundenheit abhängt. Wurde man beispielsweise nach wissenschaftlichen Vorträgen früher gefragt, warum man zu dieser oder jener Meinung gekommen sei, so lautet heute die Standardfrage: Von wem werden Sie eigentlich dafür bezahlt, daß Sie diese oder jene Meinung hier vertreten? Dieser Mechanismus führt natürlich zwangsweise dazu, daß Institutionen, bei denen eine offensichtliche

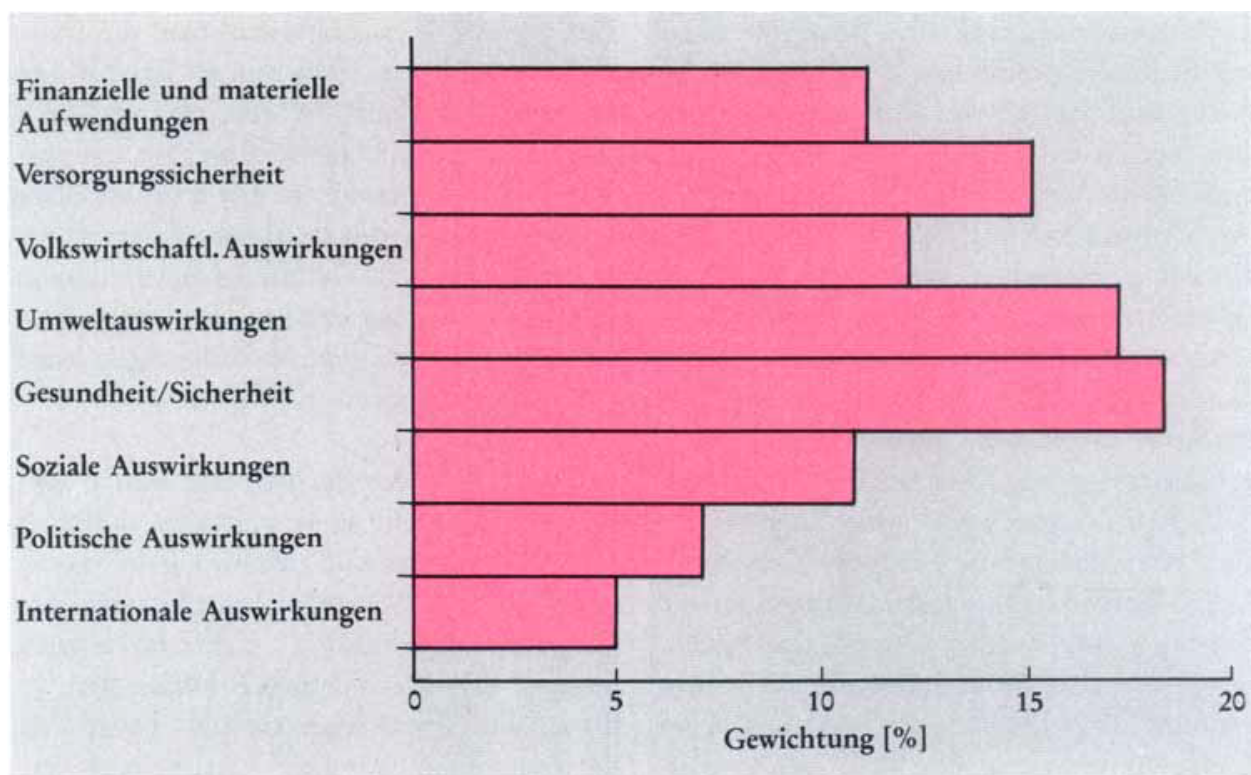

Interessengebundenheit vorliegt (z. B. die Industrie), im öffentlichen Wettstreit der Meinungen und Argumente eine schlechtere Ausgangsposition einnehmen als die Institutionen, bei denen nur latente Bindungen an Interessengruppen oder Wertgruppen vorliegen (etwa Umweltschützer).

- Die Moralisierung komplexer Sachprobleme: Je mehr Sachwissen ein bestimmtes Thema erfordert und je unübersichtlicher die Argumentationszusammenhänge rivalisierender Deutungsmuster von Technologien sind, desto schwieriger wird es für den einzelnen, sich mit dieser Materie zu beschäftigen und eine eigene Meinung oder Einstellung zu finden. Sofern er davon überzeugt ist, daß eine der beteiligten sozialen Gruppen seine Meinung gut und effektiv im Konzert der "Pressure Groups" vertritt, kann er das Thema abhaken und auf eine ihm adäquate Delegation seines Interesses hoffen. Sobald sich aber Bürger nicht mehr durch bestimmte Interessengruppen vertreten fühlen oder aber diese neue Gruppenanhänger rekrutieren wollen, muß eine Ebene der Gesprächsführung gefunden werden, die jeden nach kürzerer Vorbereitungszeit mitsprachefähig oder zumindest mithörfähig macht. Dazu eignet sich, wie der Soziologe Scheuch hervorhebt, besonders die Moralisierung von Sachproblemen. In dem Moment, wo bestimmte technische, ökonomische oder politische Sachfragen zu Fragen der Moral erhoben werden, spielt Detailwissen keine Rolle mehr (stört sogar meistens). In einem moralisierten Streit werden Punkte in der öffentlichen Debatte durch Appelle, Schuldzuweisungen, echte oder gespielte Betroffenheit und moralische Entrüstung gesammelt.
So sehr man in den fünfziger und sechziger Jahren politische und moralische Fragen durch Rekurs auf angebliche Sachzwänge von der öffentlichen Diskussion fernhalten wollte, so sehr erleben wir heute den Pendelausschlag zur anderen Seite, indem selbst die unverfänglichsten Sachfragen zu moralischen Schicksalsfragen hochstilisiert werden. Es versteht sich von selbst, daß Konflikte mit moralisierender Tiefenwirkung kaum noch durch Konsens gelöst werden können.

- Flucht in kollektive Geborgenheit: Um der Komplexität der Umwelt auszuweichen und einfachere Interpretationsmuster des Alltagsgeschehens zu entwickeln, beobachten wir in zunehmendem Maße eine Einkapselung von Individuen in Wertgruppen, die relativ homogene Interpretationsmuster bis hin zu ganzen Weltbildern anbieten. Der besondere Vorteil dieser Wertgruppen besteht darin, daß jedem Mitglied ein ganzer Satz von selbstverständlichen Überzeugungen zur Verfügung steht, der nicht nur das Wir-Gefühl in der Gruppe stärkt, sondern auch klare Lebensperspektiven für das Mitglied selbst vermittelt. In einer Welt, die durch schnellen Wertwandel und Infragestellung aller Traditionen charakterisiert ist, kommt diesen Gruppen die Funktion einer psychischen Stabilisierung und individuellen Sinnvermittlung zu. Problematisch für die notwendige Kohärenz und Integration in die Gesamtgesellschaft können solche Gruppen dann werden, wenn der Kontakt zu rivaliserenden anderen Gruppen völlig abreißt oder nur noch zum Schlagabtausch genutzt wird. Die Gefahr der Segregierung aus dem gesellschaftlichen Umfeld gilt übrigens für Banker ebenso wie für Alternativgruppen. 
Abb. 3. Mittelwerte der den einzelnen Kriterien zugemessenen Gewichte. Die nach dem Zufallsverfahren ausgewählten Bürger hatten die Aufgabe, den acht Kriterien Gewichte entsprechend der relativen Bedeutung für die Entscheidung zwischen verschiedenen Energieoptionen zuzuweisen. Die Gewichte addieren sich jeweils zu 100 Prozent.

- Die sinnstiftende Rolle von Natur in einer säkularisierten Gesellschaft: Die Säkularisierung der Gesellschaft mit der Verankerung von Wertpluralität als Kennzeichen einer liberalen Gesellschaftsordnung hat in vielen Menschen eine innere Unsicherheit, eine Suche nach Lebenssinn ausgelöst, der über den Zeithorizont des Individuums hinausreichen und universelle Geltung beanspruchen soll. Die sich ausbreitende Orientierungslosigkeit des Menschen, ausgelöst durch die Konkurrenz unterschiedlicher Wertesysteme und durch die Erkenntnis der Relativität menschlicher Ziele und Vorstellungen, hat die Sehnsucht nach einer stabilen, feststehenden und unumstößlichen Ordnung hervorgerufen. Dabei kommt der Natur eine besondere Schlüsselrolle zu. Sie verkörpert in modernen Industriegesellschaften Kontinuität, organische Entwicklungsfähigkeit und Besinnung. So wenig die Natur Antworten auf Sinnfragen oder ethische Probleme geben kann, so sehr eignet sie sich als Kristallisationspunkt für Engagement, affektive Identifikation und personale Kontinuität.

Für die Politik ergibt sich aus dieser Situation die Schwierigkeit, Offenheit gegenüber unterschiedliche Forderungen relativ abgekapselter Gruppierungen zu demonstrieren und gleichzeitig die Rationalität der eigenen Entscheidungen sicherzustellen. Der Grundkonsens demokratischer Systeme, daß sich unterlegene Minderheiten „zähneknirschend“ den Entscheidungen der Mehrheiten unterwerfen müssen, ist durch die bestehende Tendenz zur Segregierung in Wertgruppen und der fehlenden grundsätzlichen Übereinstimmung in Zielen und Verfahren staatlicher Politik gefährdet. Gleichzeitig setzt aber die Moralisierung von Sachproblemen der Möglichkeit der Politikfähigkeit durch Konsens enge Grenzen. In dieser Situation droht die politische Paralyse - Nichts geht mehr! Dabei muß zusätzlich bedacht werden, daß die verschiedenen Gruppierungen unserer Gesellschaft sehr unterschiedliche Reaktionsmuster auf politische Entscheidungen entwickelt haben. Personen, die stark umweltbezogene Werte entwickelt haben und diese durch politische Entscheidungen verletzt fühlen, setzen ihren Protest in öffentlichkeitswirksame Aktionen, wie Demonstrationen, um. Personen mit stark wirtschaftsbezogenen Werten wählen dagegen Protestformen der inneren Emigration: resignative Grundhaltungen, Leistungsverweigerungen oder sogar Kapitalflucht. In beiden Fällen entstehen der Gesellschaft soziale Kosten, die politisches Handeln notwendig machen.

\section{Technikakzeptanz als symbolische Arena für politische Konflikte}

Der sich heute abzeichnende Technologieprotest in Teilen der Bevölkerung ist nur ein Symptom für eine tieferliegende Krise des Akzeptanzverlustes kollektiv bindender Entscheidungen in der Politik. Ohne das Wort Krise überstrapazieren zu wollen, deuten viele Anzeichen in der heutigen Gesellschaft darauf hin, daß die Selbstverständlichkeit, politische Entscheidungen als notwendige Kompromißlösung in widerstreitenden Interessen zu akzeptieren, in Teilen der Bevölkerung geschwunden ist. Dabei - dies muß ausdrücklich betont werden - steht nicht das politische Entscheidungssystem als solches unter Legitimationszwang, sondern die Art, wie dieses System von den gesellschaftlichen Kräften ausgefüllt wird. Während die breite Mehrheit der Bevölkerung die demokratischen Institutionen und die Form der Prozeßsteuerung von politischen Entscheidungen als adäquat und sinnvoll betrachtet, wächst jedoch der Zweifel an der Neutralität und Kompetenz der personalen Träger dieser Institutionen. Es handelt sich also - in soziologischer Fachsprache weniger um eine Legitimationskrise des politischen Systems als um eine Implementationskrise der politischen Praxis. Die Gründe für diese Krise sind vielfältig und die wichtigsten wurden oben (Seite 49) aufgezählt.

Wir werden also nicht umhin kommen, über den Bereich der Technologiepolitik hinaus Innovationen im politischen Implementationsprozeß einzuführen. Diese Innovationen müssen darauf gerichtet sein, das System und seine Institutionen nicht grundsätzlich in Frage zu stellen, sondern die Ausführung der politischen Aufgaben zu verbessern. Dies ist keine normative Forderung, die sich aufgrund eines bestimmten Demokratieverständnisses ableiten läßt, sondern ergibt sich als praktische Konsequenz der vielfältigen empirischen Arbeiten über das Verhältnis von Bürger und Staat, die in den letzten Jahren durchgeführt worden sind.

Wie müßten die notwendigen politisch-strukturellen Innovationen beschaffen sein? Zum einen gilt es, den Distanzverlust zwischen politischem Entscheidungssystem und davon betroffenen Bürgern dadurch auszugleichen, $\mathrm{da} ß$ die Prozesse der Entscheidungsfindung und der Berücksichtigung verschiedener Interessen transparent gemacht werden; zum anderen müssen die Mitglieder des politischen Systems durch Rückkoppelungsmechanismen mit den Wünschen und Vorstellungen der Bürger vertraut gemacht werden, da der Mechanismus der Wahl und Wiederwahl als Sanktionskriterium für diese Aufgabe nicht ausreicht. Neue partizipative Formen der Einflußnahme des Bürgers auf staatliche Entscheidungen sind also gefordert, die jedoch das Gesamtsystem der Entscheidungsfindung - z. B. das der Repräsentation - nicht aufweichen dürfen.

Dennoch ist davor zu warnen, Partizipation als Allheilmittel zur Lösung politischer Probleme aufzufassen. Mitwirkung und Mitbestimmung von Bürgern an der politischen Entscheidungsfindung können zunächst einmal Zielkonflikte nicht aus der Welt schaffen und Interessengegensätze nicht auflösen. Dazu einige kurze Stichworte:

- Die Forderung nach Betroffenen-Beteiligung ist in einer Gesellschaft mit hochdifferenzierter Arbeitsteilung illusorisch. Denn wer betroffen ist und wer nicht, läßt sich aufgrund der in sich verflochtenen Wirtschaftsstruktur nicht mehr eindeutig ausmachen. Beim Bau eines Kraftwerkes läßt sich beispielsweise nicht entscheiden, in welcher Weise die Stromnutzer als Betroffene zu Wort kommen sollen. Von den Umweltauswirkungen des Kraftwerks sind auch nicht nur die Anrainer betroffen, sondern möglicherweise auch andere Personen in weit entfernten Landstrichen. Das gerade aktuelle Problem des Waldsterbens gibt ja ein beredtes Zeugnis dafür. Auch die in den sechziger Jahren praktizierte Politik des Baus von hohen Schornstei- 
nen zur gleichmäßigeren Verteilung von umweltschädlichen Stoffen ist ein gutes Beispiel dafür, daß unmittelbare Nachbarn von technischen Anlagen eine Minderung ihres Risikos auf Kosten der Allgemeinheit vornehmen können.

- Die Volksmeinung ist keineswegs einheitlich. Mögen auch die in einer Bürgerinitiative versammelten Mitglieder alle eine bestimmte Lösung der Problematik bevorzugen, so gibt es wiederum andere Gruppen, die meist schwerer zu organisieren sind, denen eine andere Lösung lieber wäre. In dem Moment aber, indem Bürgerinitiativen Mitwirkungsrechte erhielten, ist es nach den Erkenntnissen der Logik kollektiven Handelns wahrscheinlich, daß Gegenbürgerinitiativen entstehen, die ihre Interessen gegenüber den anderen Bürgerinitiativen durchsetzen möchten. Trotz Partizipation wird man nicht umhin können, Konflikte zugunsten der einen oder anderen Seite exemplarisch zu lösen.

- Ein drittes schwerwiegendes Problem ist die Frage der Kontinuität von Entscheidungen. Wenn Partizipation von Bürgern Sinn machen soll, dann kann es sich nicht um repräsentativ gewählte Gremien handeln, die über eine längere Mandatsperiode Mitsprache- und Mitwirkungsrechte beanspruchen. Wenn es sich aber nur um punktuelle Einflußnahmen auf bestimmte Entscheidungen handelt, wird es sehr schwierig sein, positive oder negative Lerneffekte bei der Implementierung der Entscheidungen zu berücksichtigen. Ebenfalls fehlt bei partizipativ getroffenen Entscheidungen ein direkter Sanktionsmechanismus für offenkundige Fehlleistungen: Weder die Sanktion von Wahl und Wiederwahl noch rechtliche Schritte können gegen eine Entscheidung beteiligter Bürger vorgenommen werden.

Mit den obigen Ausführungen soll nicht der Eindruck erweckt werden, als ob Bürgerbeteiligung politisch unfruchtbar sei. Im Gegenteil: Die Stabilität unseres politischen Systems wird in Zukunft weitgehend davon abhängen, daß das politische System mehr auf die Präferenzen und Wünsche der Bürger eingeht. Nur muß Partizipation so geschickt in das System integriert werden, daß die Vorteile des parlamentarischen Entscheidungssystems nicht auBer Kraft gesetzt werden. Bis heute fehlen uns noch schlüssige Konzepte, um dieses Ziel zu verwirklichen. Vieles, was heute in dieser Hinsicht vorgeschlagen wird, ist meines Erachtens politisch unpraktikabel oder sogar gefährlich.
Einige Vorschläge sollten jedoch die Chance einer Bewährungsprobe erhalten.

Eine erfolgversprechende Möglichkeit ist z. B. das Konzept der Planungszelle, das von dem Wuppertaler Sozialwissenschaftler Peter Dienel entwickelt worden ist. Bei den Planungszellen handelt es sich um Gruppen von zufällig ausgewählten Bürgern, die exemplarisch für die Gesamtheit Planungsaufgaben bearbeiten und Empfehlungen an die Entscheidungsträger weiterleiten sollen. Dadurch können Wünsche, Präferenzen und Wertverschiebungen frühzeitig an die offizielle Politik weitergeleitet und in die Gewichtung von Handlungsalternativen eingehen. Die Programmgruppe "Technik und Gesellschaft" in der Kernforschungsanlage Jülich hat beispielsweise in Zusammenarbeit mit Professor Dienel und seinen Mitarbeitern in ganz Deutschland im Bereich der Energiepolitik 24 derartige Planungszellen organisiert, um die Wünsche und Vorstellungen der Bürger zu einer sozialverträglichen Energiepolitik der Zukunft auszuloten. Das Ergebnis war so ermutigend, daß es sich lohnt, auf diesem Weg weiter fortzufahren.

\section{Literatur}

G. Bechmann, G. Frederichs und H. Paschen: "Risikoakzeptanz und Wertwandel". Zeitschrift für Angewandte Systemanalyse 1981, Heft 2, S. 199 ff.

Bundesminister für Forschung und Technologie (Hrsg.): „Politik, Wertwandel, Technologie. Ansichtspunkte für eine Theorie der sozialen Entwicklung." Düsseldorf, Wien 1982.

P. C. Dienel: „Die Planungszelle“. Opladen 1977.

H. Jungermann: ,Zur Wahrnehmung und Akzeptierung des Risikos von Großtechnologien." Psychologische Rundschau 1982, Heft 23, S. $217 \mathrm{ff}$.

H. Klages und P. Kmieciak (Hrsg.): „Wertwandel und gesellschaftlicher Wandel". Frankfurt a. M. 1979.

A. Mazur: „Opposition of Technological Innovation“. Minerva 1975, Heft 13, S. 58 ff.

H. Otway: „Perception and Acceptance of Risks“. Zeitschrift für Umweltpolitik 1980, Heft 2.
H. Otway und D. von Winterfeldr: „Beyond acceptable risk: On the social acceptability of technologies". Policy Sciences 1982, Heft 14, S. $247 \mathrm{ff}$.

O. Renn: „Risikowahrnehmung der Kernenergie“. Frankfurt a. M., New York 1984.

O. Renn, G. Albrecht, U. Kotte, H. P. Peters und H. U. Stegelmann: „Sozialverträgliche Energiepolitik“. Ein Gutachten für die Bundesregierung. München 1985.

E. Swaton und O. Renn: „Attitudes towards Nuclear Power. A Comparison between Three Nations." Working Paper WP-84-11. International Institute for Applied Systems Analysis (IIASA), Laxenburg 1983.

A. Wünschmann: „Unbewußt dagegen. Zur Psychologie der Kernenergiekontroverse.“ Bonn aktuell, Stuttgart 1984.

Photo auf S. 44: SZ-Archiv.

Dr. rer. pol. Dipl.-Volkswirt O. Renn, geb. 1951. Studium der Volkswirtschaftslehre und Soziologie an der Universität Köln, Zusatzausbildung für den Fachbereich Journalismus an der Kölner Schule, Institut für Publizistik, Rodenkirchen; Promotion im Fach Sozialpsychologie. Seit 1977 wissenschaftlicher Mitarbeiter der Kernforschungsanlage Jülich, seit 1981 Leiter der Abteilung Mensch und Technik in der Programmgruppe Technik und Gesellschaft; Lehrbeauftragter für Techniksoziologie an der Universität Stuttgart. Arbeitsschwerpunkte: Technikfolgenabschätzung, Einstellungsforschung, Techniksoziologie, Entscheidungstheorie und Partizipationsforschung. Autor mehrerer Bücher, u.a. „Die sanfte Revolution, Zukunft ohne Zwang“ (1980); „Technik auf dem Prüfstand, Methoden und Maßstäbe der Technologie-Bewertung" (mit E. Münch und T. Roser, 1982); ,Risikowahrnehmung der Kernenergie" (1984); „Die Wertbaumanalyse. Entscheidungshilfe für die Politik" (mit R. Keeney, D. von Winterfeldt und U. Kotte, 1984), „Sozialverantwortliche Energiepolitik" (mit H. P. Peters, v. Albrecht, V. Kolk und H. V. Stegelmann). 